\title{
Weakly Bound $H$ Dibaryon from SU(3)-Flavor-Symmetric QCD
}

\author{
Jeremy R. Green $\odot^{*}, \dagger$ \\ Theoretical Physics Department, CERN, 1211 Geneva 23, Switzerland \\ Andrew D. Hanlon $\oplus^{*}$ \\ Helmholtz-Institut Mainz, Johannes Gutenberg-Universität, 55099 Mainz, Germany; \\ GSI Helmholtzzentrum für Schwerionenforschung, 64291 Darmstadt, Germany; \\ and Physics Department, Brookhaven National Laboratory, Upton, New York 11973, USA \\ Parikshit M. Junnarkar ${ }^{\S}$ \\ Institut für Kernphysik, Technische Universität Darmstadt, Schlossgartenstraße 2, 64289 Darmstadt, Germany \\ Hartmut Wittig (1) $\|$ \\ PRISMA Cluster of Excellence and Institut für Kernphysik, University of Mainz, Becher Weg 45, D-55099 Mainz, Germany; \\ Helmholtz-Institut Mainz, Johannes Gutenberg-Universität, 55099 Mainz, Germany; \\ and GSI Helmholtzzentrum für Schwerionenforschung, 64291 Darmstadt, Germany
}

(Received 10 March 2021; revised 12 October 2021; accepted 12 November 2021; published 9 December 2021)

\begin{abstract}
We present the first study of baryon-baryon interactions in the continuum limit of lattice QCD, finding unexpectedly large lattice artifacts. Specifically, we determine the binding energy of the $H$ dibaryon at a single quark-mass point. The calculation is performed at six values of the lattice spacing $a$, using $\mathrm{O}(a)$ improved Wilson fermions at the $\mathrm{SU}(3)$-symmetric point with $m_{\pi}=m_{K} \approx 420 \mathrm{MeV}$. Energy levels are extracted by applying a variational method to correlation matrices of bilocal two-baryon interpolating operators computed using the distillation technique. Our analysis employs Lüscher's finite-volume quantization condition to determine the scattering phase shifts from the spectrum and vice versa, both above and below the two-baryon threshold. We perform global fits to the lattice spectra using parametrizations of the phase shift, supplemented by terms describing discretization effects, then extrapolate the lattice spacing to zero. The phase shift and the binding energy determined from it are found to be strongly affected by lattice artifacts. Our estimate of the binding energy in the continuum limit of three-flavor QCD is $B_{H}^{\mathrm{SU}(3)_{f}}=4.56 \pm 1.13_{\text {stat }} \pm 0.63_{\text {syst }} \mathrm{MeV}$.
\end{abstract}

DOI: 10.1103/PhysRevLett.127.242003

The $H$ dibaryon is a scalar six-quark state with flavor content $u u d d s s$ originally proposed in 1977 by Jaffe [1]. Despite years of effort, experimental searches have not produced any hard evidence for its existence [2-4]. However, an upper bound on its binding energy has been derived from the observed production and decay pattern of a doubly strange ${ }_{\Lambda \Lambda}^{6} \mathrm{He}$ hypernucleus $[2,3]$.

Studying the properties of a potential $\Lambda-\Lambda$ bound state will help our understanding of the hadronic $(\Lambda-\Lambda)$ interaction, which is relevant for the physics of double hypernuclei, neutron-rich matter, and neutron stars. Recently, experimental data for two-particle correlations in $p$ - $p$, $p-\mathrm{Pb}$, and $\mathrm{Au}-\mathrm{Au}$ collisions [5-7] have been analyzed to

Published by the American Physical Society under the terms of the Creative Commons Attribution 4.0 International license. Further distribution of this work must maintain attribution to the author(s) and the published article's title, journal citation, and DOI. Funded by SCOAP . constrain the $\Lambda-\Lambda$ interaction and provide model estimates for the binding energy of the $H$ dibaryon. In addition, a dedicated experiment is planned to search for it at J-PARC [8]. Other approaches to study the $H$ dibaryon include chiral effective field theory [9-12] and lattice QCD.

Lattice QCD studies of dibaryons and baryon-baryon scattering are very challenging because of the signal-tonoise problem $[13,14]$ and the complexity of contractions. In response to an inconsistency among results in the nucleon-nucleon sector $[15,16]$, there has been a recent focus on improved baryon-baryon spectroscopy methods [17-19]. This work goes beyond that to achieve control over all systematic effects for the $H$-dibaryon channel at one unphysical quark-mass point.

There is a long history of calculations studying whether the $H$ dibaryon is a prediction of QCD [17,20-34]. Results for the binding energy $B_{H}$ from these calculations vary considerably, with estimates ranging from a few $\mathrm{MeV}$ up to $75 \mathrm{MeV}$, depending on the methodology and/or the value of the pion mass (see Fig. 5). Recently, employing 
near-physical pion and kaon masses, the HAL QCD Collaboration reported that the $\Lambda-\Lambda$ interaction is only weakly attractive and does not sustain a bound or resonant dihyperon [34].

In our previous work [17], using gauge fields with dynamical $u$ and $d$ quarks and a quenched $s$ quark, we found that the distillation method [35] produced a better determination of the two-baryon spectrum than previously used methods. At a heavy SU(3)-symmetric point with a pion mass of $960 \mathrm{MeV}$, we obtained $B_{H}=19 \pm 10 \mathrm{MeV}$.

In this Letter, we extend our calculations to lattice QCD with dynamical $u, d$, and $s$ quarks with degenerate masses set to their physical average value corresponding to $m_{\pi}=$ $m_{K} \approx 420 \mathrm{MeV}$ [36]. We present the first systematic study of discretization effects in a multibaryon system by computing finite-volume spectra at several lattice spacings, extrapolating the corresponding scattering phase shift to the continuum limit, and determining the binding energy. As shown in Fig. 1, at vanishing lattice spacing, we find $B_{H}^{\mathrm{SU}(3)_{f}}=4.56 \pm 1.30 \mathrm{MeV}$, which is smaller than the result at the coarsest lattice spacing by a factor of about 7.5. We conclude that a thorough investigation of lattice artifacts is indispensable for answering the question whether a bound $H$ dibaryon exists in nature.

Our calculations are based on a set of eight gauge ensembles generated by CLS [38], with a nonperturbatively $\mathrm{O}(a)$-improved Wilson-clover fermion action. These ensembles have six different values of the lattice spacing and multiple box sizes $L$ (all satisfying $m_{\pi} L \geq 4.4$ ) as shown in the inset of Fig. 3 [39].

For each ensemble, we determine the energy levels in the rest frame and in four moving frames. To this end, in each frame we compute a Hermitian matrix of two-point correlation functions from a basis of interpolating operators: $C_{i j}(t) \equiv\left\langle\mathcal{O}_{i}(t) \mathcal{O}_{j}^{\dagger}(0)\right\rangle$. The finite-volume spectrum $\left\{E_{n}\right\}$ determines the exponential falloff of $C_{i j}(t)$.

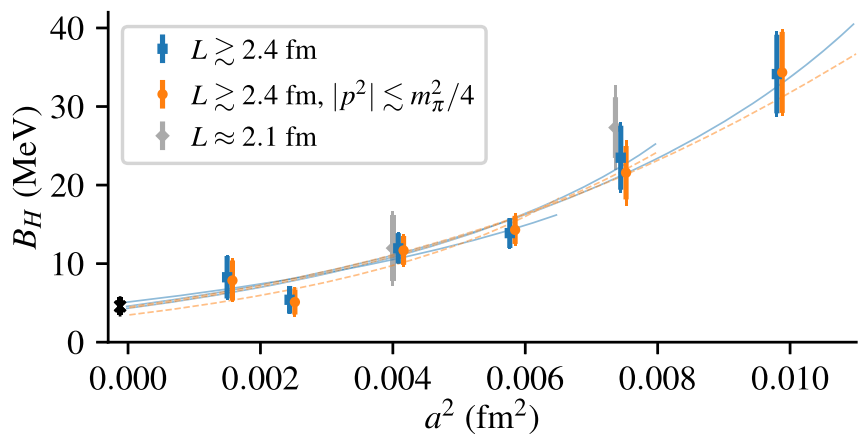

FIG. 1. Binding energy versus squared lattice spacing from fits to the full $p^{2}$ range (blue squares and solid curves) and to the near-threshold region (orange circles and dashed curves). Points are from fits to individual ensembles and curves are from the combined fits to the spectra of different subsets of the ensembles; they are not fitted to these points. Gray diamonds show results from the small-volume ensembles and the black cross shows our final estimate.
The building blocks of our operator basis are products of two single-baryon operators projected to momenta $\vec{p}_{1}$ and $\vec{p}_{2}$ with total spin zero or one. For each frame momentum $\vec{P}=\vec{p}_{1}+\vec{p}_{2}$, we take linear combinations that transform under the trivial irreducible representation of the little group of $\vec{P}$, which contains the ${ }^{1} S_{0}$ scattering channel [39]. Following Refs. [31,52], the flavor content of our interpolating operators is a linear combination of isospinzero $\Lambda \Lambda, \Sigma \Sigma$, and symmetric $N \Xi$ that corresponds to the singlet irreducible representation of SU(3) flavor.

Calculating the correlation functions of bilocal operators requires the ability to compute "time-slice-to-all" quark propagators. As in our previous study [17], we have used the distillation technique $[35,39]$.

The finite-volume energy levels in each frame are determined by solving a generalized eigenvalue problem $[39,53,54], C\left(\tau_{D}\right) v_{n}=\lambda_{n} C\left(\tau_{0}\right) v_{n}$ for fixed $\tau_{0}$ and $\tau_{D}$ satisfying $\tau_{D}>\tau_{0} \geq \tau_{D} / 2$. We then use the eigenvectors $v_{n}$ to construct $\tilde{C}_{n m}(t) \equiv v_{n}^{\dagger} C(t) v_{m}$, an approximately diagonalized correlator matrix. We have verified that different combinations of $\left(\tau_{0}, \tau_{D}\right)$ yield consistent results across a wide range of values [39].

Before fitting to the data, we divide the rotated twobaryon correlators by a product of two single-baryon correlators that form the corresponding two-baryon noninteracting level $R_{n}(t) \equiv \tilde{C}_{n n}(t) /\left[C_{\Lambda}^{\vec{p}_{1}}(t) C_{\Lambda}^{\vec{p}_{2}}(t)\right]$, where $C_{\Lambda}^{\vec{p}_{i}}$ is a single- $\Lambda$ correlator with momentum $\vec{p}_{i}$, and the total frame momentum is $\vec{p}_{1}+\vec{p}_{2}$. The leading term in this ratio falls off exponentially with the shift $\Delta E$ of the interacting two-baryon energy away from the noninteracting level. In the ratio, we observe a partial cancellation of correlated statistical fluctuations and residual contributions from excited states, which helps in the reliable determination of $\Delta E$.

Our finite-volume energies are determined from singleexponential fits to $R_{n}(t)$. For all levels, we choose $t_{\min }$, i.e., the smallest time separation included in the fits, to lie in the plateau region of $R_{n}(t)$. We also aim to have $t_{\min }$ lie in the plateau region of the single-baryon correlators, and in the majority of cases we set it to be the first time separation in this plateau region. Since the single-baryon correlators take longer than the two-baryon correlators to reach their asymptotic behavior, this ensures that all correlators entering the ratio have little to no excited-state contamination. In some cases, however, the signal of $R_{n}(t)$ is already significantly degraded at the start of the single-baryon plateau region, and we are led to choose a slightly lower $t_{\min }$ that still lies within the plateau region of the correlator ratio. For all levels, we estimate the sensitivity to $t_{\min }$ by extracting an alternative spectrum with $t_{\min }$ further lowered, and use it in subsequent analyses to estimate the systematic uncertainty of our energy determination.

The fits also yield the couplings between each energy eigenstate and our operators. For each frame that includes a spin-one operator, we find one eigenstate that has strong 


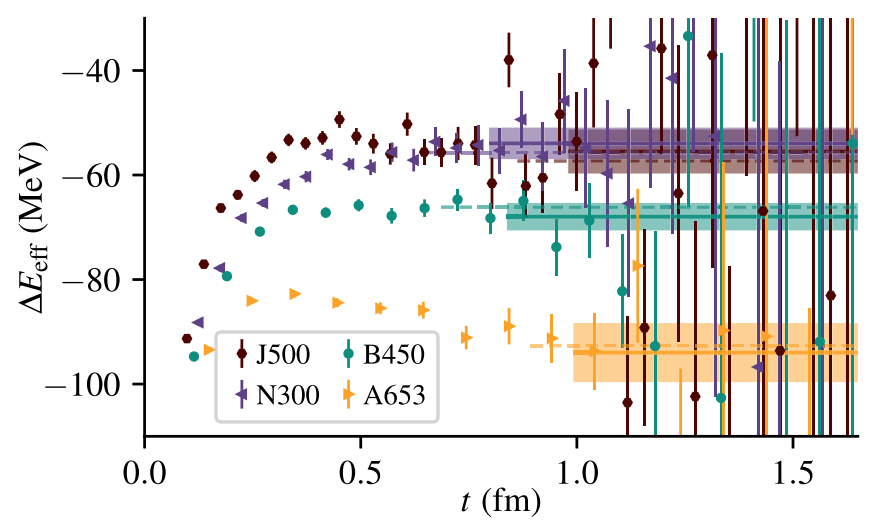

FIG. 2. Effective energy difference obtained from $R_{0}(t)$ for the ground state in frame $(0,0,1)$ on four ensembles with similar volumes. The bands show $\Delta E$ determined from a single-exponential fit to $R_{0}(t)$ and also indicate the range of $t$ used for the fit. The dashed lines show the alternative fit used to estimate systematic uncertainty.

overlap with only that operator, allowing for a simple identification of the spin-one dominated states.

Figure 2 shows the effective energy difference $\Delta E_{\text {eff }}(t) \equiv-(d / d t) \log R(t)$ and the extracted $\Delta E$ for the ground state in frame $(0,0,1)$ on four ensembles that differ primarily in their lattice spacing. This level is particularly important because it is the closest to the bound-state pole determined in the phase shift analysis.
An overview of the finite-volume spectrum is shown in Fig. 3, where the energy shifts are transformed to the center-of-mass momentum $p$. For every level, these two figures show a clear increasing trend as the lattice spacing is reduced, indicating that discretization effects are significant.

Given the two-particle scattering amplitude, Lüscher's finite-volume quantization condition [55] and its generalizations [56-58] determine the finite-volume spectrum, up to exponentially suppressed corrections, between the $t$-channel cut $\left(p^{2}>-m_{\pi}^{2} / 4\right)$ and the three-particle threshold $\left(E_{\mathrm{c} . \mathrm{m} .}<2 m_{B}+m_{\pi}\right)$. Since the quantization condition is diagonal in spin [57,58], the spin-one part of the scattering amplitude does not affect the spin-zero finitevolume spectrum, and we choose to ignore the spin-one states. In addition, we neglect higher partial waves starting from ${ }^{1} D_{2}$. In this case, the quantization condition yields the ${ }^{1} S_{0}$ phase shift $\delta(p)$ at the momentum corresponding to each finite-volume energy level:

$$
p \cot \delta(p)=\frac{2}{\sqrt{\pi} L \gamma} Z_{00}^{\vec{P} L /(2 \pi)}\left(1,\left(\frac{p L}{2 \pi}\right)^{2}\right),
$$

where $\gamma=E / E_{\text {c.m. }}$ and $Z_{00}^{\vec{D}}$ is a generalized zeta function. In addition to excluding levels with too-low or too-high $p^{2}$ from our analysis, we must also exclude the first excited levels in frames $(0,1,1)$ and $(1,1,1)$, as the ${ }^{1} D_{2}$ partial wave

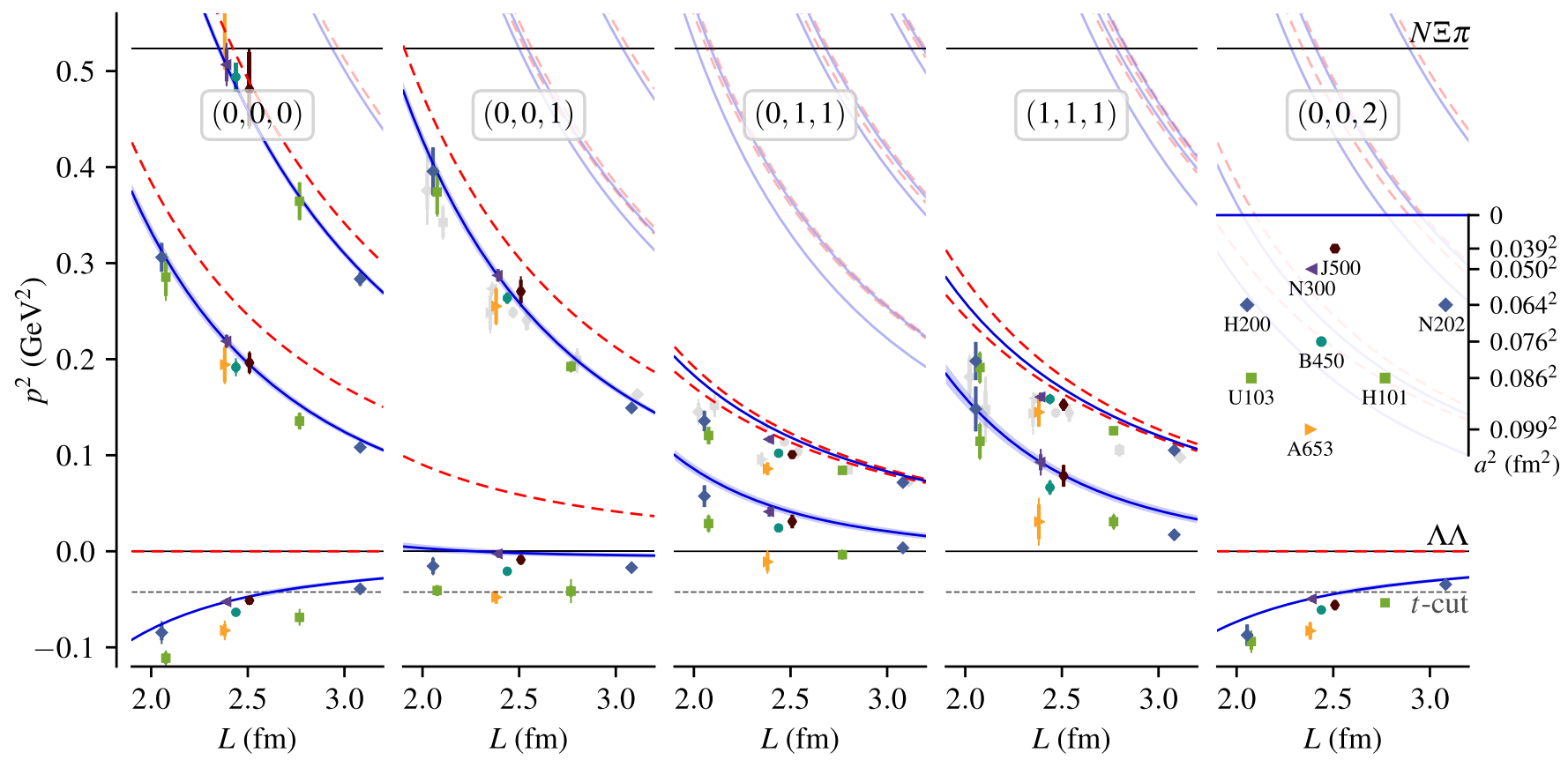

FIG. 3. Finite-volume spectrum: center-of-mass scattering momentum $p^{2}$ versus lattice extent $L$. The five different frames are shown separately and are labeled with $\vec{D} \equiv \vec{P} L /(2 \pi)$. Colored points show spin-zero levels, and gray points (offset horizontally) show levels identified as spin-one. Solid horizontal lines show the two- and three-particle thresholds, while dashed horizontal lines represent the $t$-channel cut. The noninteracting spectrum is denoted by red dashed curves, and solid blue curves show the interacting spectrum determined in the continuum (see main text); the pale curves correspond to levels that have not been determined in the lattice calculation. The inset serves as a legend showing $L$ and $a^{2}$ for the ensembles used in this Letter. 
is necessary to describe their position below the lowest noninteracting level [39].

The quantization conditions do not take discretization effects into account; strictly speaking, they are only valid in the continuum. There is no general formalism for finitevolume quantization at nonzero lattice spacing, except for a simple model studied in Ref. [59]. In principle, discretization effects would affect both the scattering amplitude and the finite-volume quantization condition. Effects on the former could include $a$ dependence and frame dependence of the scattering amplitude, as well as couplings between $J^{P}$ that are forbidden in the continuum. Effects on the latter could include a modification of the zeta functions [59]. Either way, discretization effects might spoil the factorization that separates spin-zero from spin-one. Lacking a rigorous understanding, we have elected to model discretization effects in a simple way by allowing the parameters of the phase shift to depend on $a$.

Our primary analysis is based on combined fits of the dependence of the phase shift on both $p^{2}$ and $a$. Specifically, our model is

$$
p \cot \delta(p)=\sum_{i=0}^{N-1} c_{i} p^{2 i}, \quad c_{i}=c_{i 0}+c_{i 1} a^{2} .
$$

Concerning the dependence on $p^{2}$, we fit in two ways. The first uses the near-threshold region $\left|p^{2}\right| \lesssim m_{\pi}^{2} / 4$ (where the effective range expansion converges) with $N=2$ terms for the dependence on $p^{2}$. The second uses the full $p^{2}$ range, starting from the $t$-channel cut and stopping just below the three-particle threshold, with $N=3$. Given $\left\{c_{i j}\right\}$, solving Eq. (1) yields a discrete spectrum of $p^{2}$ for each volume and frame; we fit these to the lattice spectra. For comparison, we also performed fits to individual ensembles, neglecting discretization effects. Given $\delta(p)$, a solution below threshold to $p \cot \delta(p)=-\sqrt{-p^{2}}$ corresponds to a bound-state pole. All of the fits yielded a bound $H$ dibaryon.

Our preferred fit is to all ensembles using the full $p^{2}$ range; the corresponding continuum interacting energy levels are shown as blue curves in Fig. 3. In addition to the alternative spectrum fit range, we estimate the systematic uncertainty using the root-mean-square difference of alternative combined fits that cover all combinations of cuts on $p^{2}$ (full range or near threshold), $a$ (all six or the finest four), and $L$ (excluding $L \approx 2.1 \mathrm{fm}$ or not). All of these fits have acceptable fit quality, with $p$ values between 0.2 and 0.9. We explored adding an $a^{3}$ term in Eq. (2) but found that this reduces $\chi^{2}$ by at most 1.1 for each additional fit parameter, a sign of overfitting.

The phase shifts from the preferred fit in the continuum and at nonzero lattice spacing corresponding to the four ensembles with $L \approx 2.4 \mathrm{fm}$ (J500, N300, B450, A653) are shown in Fig. 4. Since these ensembles have similar values of $L$, they allow us to perform a cross-check shown in the

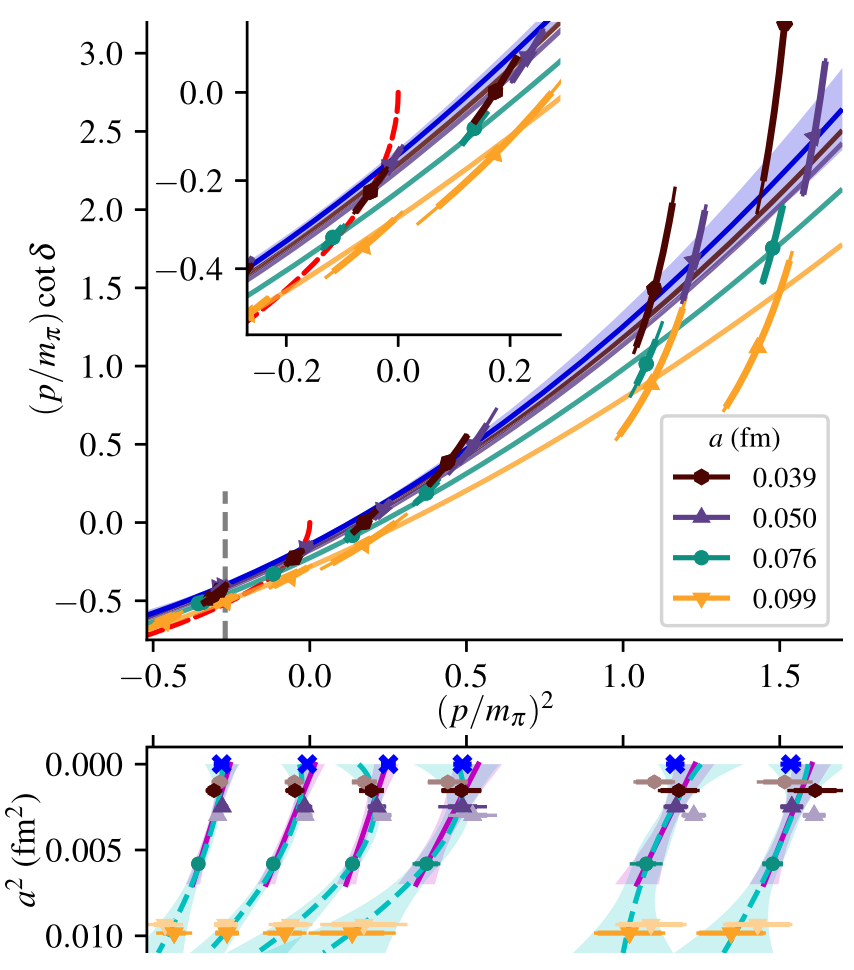

FIG. 4. Upper panel: $p \cot \delta$ versus $p^{2}$ normalized using the pion mass, with inset showing the near-threshold region. Data are shown for the four ensembles with $L \approx 2.4 \mathrm{fm}$. Curves show the result from a combined fit at nonzero lattice spacing (indicated by color) and in the continuum (blue with error band); intersections with the red dashed curve correspond to bound-state poles. Only points to the right of the vertical dashed line are included in the fit. Lower panel: level-by-level cross-check of continuum extrapolation, with adjustments on three ensembles to match the target volume $L^{*}$. Pale points (displaced vertically) show the levels before adjustment. The spectrum obtained from the continuum phase shift is indicated using blue crosses. Curves show continuum extrapolations of the form $b_{0}+b_{1} a^{2}$ excluding the coarsest lattice spacing (solid magenta) and $b_{0}+b_{1} a^{2}+b_{2} a^{3}$ using all four lattice spacings (dashed cyan).

lower panel. We select the volume of ensemble B450 as our target and call this box size $L^{*}$. For the three other lattice spacings, we estimate each energy level at $L^{*}$ by shifting from $L$ using the quantization condition: $p^{2}\left(L^{*}\right) \approx p^{2}(L)+$ $p_{\mathrm{QC}}^{2}\left(L^{*}\right)-p_{\mathrm{QC}}^{2}(L)$. For each energy level, we then study the dependence of $p^{2}\left(L^{*}\right)$ on the lattice spacing and compare it with the value obtained from applying the quantization condition to the continuum limit of the preferred fit. The cross-check shows that a level-by-level continuum extrapolation at $L^{*}$ is consistent with the latter. However, some levels show curvature in the dependence on $a^{2}$ and the fixed- $L^{*}$ extrapolation is less precise, making it less useful than the combined fits.

Near threshold, we can write $p \cot \delta=-1 / a_{0}+$ $r_{0} p^{2} / 2+O\left(p^{4}\right)$, where $a_{0}$ is the scattering length and $r_{0}$ is the effective range. We obtain 


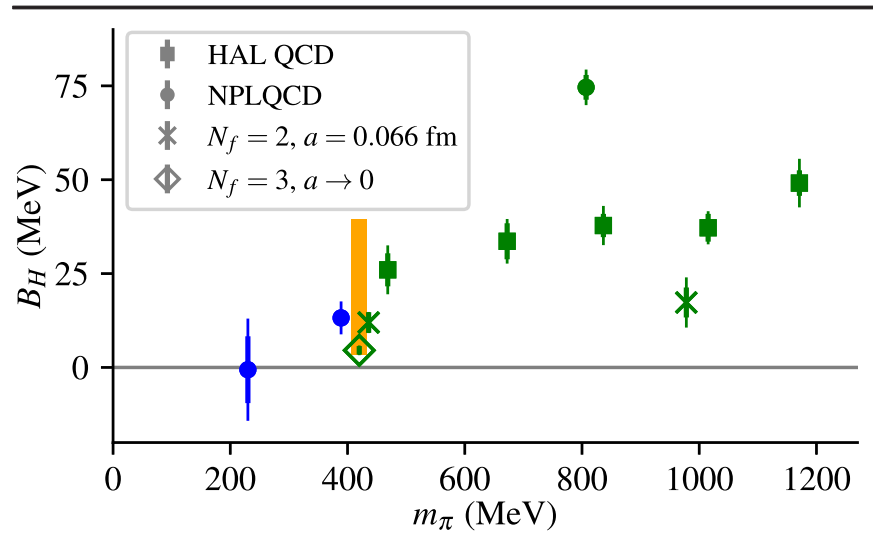

FIG. 5. Binding energy versus pion mass: our results - threeflavor QCD in the continuum [diamond, Eq. (5)] and two-flavor QCD at nonzero lattice spacing (crosses [39]) — and published values [27,28,30,32,33]. Green and blue symbols refer to SU(3)symmetric and broken cases, respectively. The orange vertical band represents the range of binding energies obtained at nonzero lattice spacing for $N_{f}=3$.

$$
\begin{aligned}
& a_{0}^{\mathrm{SU}(3)_{f}}=3.30 \pm 0.36 \pm 0.21 \mathrm{fm}, \\
& r_{0}^{\mathrm{SU}(3)_{f}}=0.98 \pm 0.04 \pm 0.05 \mathrm{fm},
\end{aligned}
$$

where the first error is statistical and the second is systematic. The dependence of the $H$ dibaryon binding energy on $a$ is shown in Fig. 1; in the continuum, we obtain

$$
B_{H}^{\mathrm{SU}(3)_{f}}=4.56 \pm 1.13 \pm 0.63 \mathrm{MeV},
$$

which is substantially lower than the binding energies determined at nonzero lattice spacing, except on the finest two of our ensembles.

We report the first lattice study of a baryon-baryon system in the continuum limit. The crucial elements of our methodology are the finite-volume quantization condition supplemented by terms describing discretization effects and applied over a wide range of lattice spacings, as well as the subsequent extrapolation to the continuum limit. We conclude that cutoff effects are large and cannot be ignored in an investigation of the $H$ dibaryon using lattice QCD; it will be essential to study their importance in other multibaryon systems such as the deuteron, where calculations disagree $[15,16,18]$. Our final result for the binding energy given in Eq. (5) suggests the existence of a weakly bound $H$ dibaryon, which is not only at variance with Jaffe's original bag model prediction [1] of a deeply bound uuddss state, but is also substantially lower than the binding energies determined in previous lattice calculations [17,27-33] at nonzero lattice spacing (see Fig. 5). This adds to the evidence against deeply bound hexaquark dark matter [60-67]. An obvious caveat is that our calculation was performed for one set of degenerate quark masses. The issue of SU(3) symmetry breaking, which is crucial, since the splitting between physical $\Lambda \Lambda$ and $N \Xi$ thresholds is larger than $B_{H}^{\mathrm{SU}(3)_{f}}$, is currently under investigation [68]. Previous estimates based on extrapolations of lattice data found a bound state at the physical point unlikely $[9,11,33,69,70]$; our smaller binding energy should make it even less likely.

We thank Maxwell T. Hansen, Ben Hörz, and Daniel Mohler for many helpful conversations. Calculations for this project used resources on the supercomputers JUQUEEN [71], JURECA [72], and JUWELS [73] at Jülich Supercomputing Centre. The authors gratefully acknowledge the support of the John von Neumann Institute for Computing and Gauss Centre for Supercomputing e.V. for Project No. HMZ21. The raw distillation data were computed using QDP++ [74], PRIMME [75], and the deflated SAP+ GCR solver from OPENQCD [76]. Contractions were performed with a high-performance BLAS library using the PYTHON package OPT_EINSUM [77]. The correlator analysis was done using SIGMOND [78]. Much of the data handling and the subsequent phase shift analysis was done using NUMPY [79] and SCIPY [80]. The plots were prepared using MATPLOTLIB [81]. This research was partly supported by Deutsche Forschungsgemeinschaft (DFG, German Research Foundation) through the Cluster of Excellence "Precision Physics, Fundamental Interactions and Structure of Matter" (PRISMA+ EXC 2118/1) funded by the DFG within the German Excellence Strategy (Project ID No. 39083149), as well as the Collaborative Research Centers SFB 1044 "The low-energy frontier of the Standard Model" and CRC-TR 211 "Strong-interaction matter under extreme conditions" (Project ID No. 315477589-TRR 211). A. D. H. is supported by the U.S. Department of Energy, Office of Science, Office of Nuclear Physics by Contract No. DESC0012704 (S. M.) and the U.S. Department of Energy, Office of Science, Office of Nuclear Physics and Office of Advanced Scientific Computing Research, within the framework of Scientific Discovery through Advance Computing Award Computing the Properties of Matter with Leadership Computing Resources. We are grateful to our colleagues within the CLS initiative for sharing ensembles.

*green@maths.tcd.ie

Present address: School of Mathematics and Hamilton Mathematics Institute, Trinity College, Dublin 2, Ireland. *ahanlon@bnl.gov

\$parikshit@theorie.ikp.physik.tu-darmstadt.de "hartmut.wittig@uni-mainz.de

[1] R. L. Jaffe, Perhaps a Stable Dihyperon, Phys. Rev. Lett. 38, 195 (1977); 38, 617(E) (1977).

[2] H. Takahashi et al., Observation of a ${ }_{\Lambda \Lambda}^{6} \mathrm{He}$ Double Hypernucleus, Phys. Rev. Lett. 87, 212502 (2001). 
[3] J. Ahn et al. (E373 (KEK-PS) Collaboration), Double- $\Lambda$ hypernuclei observed in a hybrid emulsion experiment, Phys. Rev. C 88, 014003 (2013).

[4] B. Kim et al. (Belle Collaboration), Search for an $H$-Dibaryon with Mass near $2 m_{\Lambda}$ in $\Upsilon(1 S)$ and $\Upsilon(2 S)$ Decays, Phys. Rev. Lett. 110, 222002 (2013).

[5] L. Adamczyk et al. (STAR Collaboration), $\Lambda \Lambda$ Correlation Function in $\mathrm{Au}+\mathrm{Au}$ Collisions at $\sqrt{s_{\mathrm{NN}}}=200 \mathrm{GeV}$, Phys. Rev. Lett. 114, 022301 (2015).

[6] S. Acharya et al. (ALICE Collaboration), $p-p, p-\Lambda$, and $\Lambda-\Lambda$ correlations studied via femtoscopy in $p p$ reactions at $\sqrt{s}=7$ TeV, Phys. Rev. C 99, 024001 (2019).

[7] S. Acharya et al. (ALICE Collaboration), Study of the $\Lambda-\Lambda$ interaction with femtoscopy correlations in $p p$ and $p$-Pb collisions at the LHC, Phys. Lett. B 797, 134822 (2019).

[8] Y. Ichikawa et al. (J-PARC E42, 45, 72 Collaborations), Time projection chamber "HypTPC" for the hadron spectroscopy at J-PARC, Phys. Soc. Jpn. Conf. Proc. 33, 011103 (2021).

[9] J. Haidenbauer and U.-G. Meißner, To bind or not to bind: The $H$-dibaryon in light of chiral effective field theory, Phys. Lett. B 706, 100 (2011).

[10] J. Haidenbauer, U.-G. Meißner, and S. Petschauer, Strangeness $S=-2$ baryon-baryon interaction at next-to-leading order in chiral effective field theory, Nucl. Phys. A954, 273 (2016).

[11] K.-W. Li, T. Hyodo, and L.-S. Geng, Strangeness $S=-2$ baryon-baryon interactions in relativistic chiral effective field theory, Phys. Rev. C 98, 065203 (2018).

[12] V. Baru, E. Epelbaum, J. Gegelia, and X. L. Ren, Towards baryon-baryon scattering in manifestly Lorentz-invariant formulation of SU(3) baryon chiral perturbation theory, Phys. Lett. B 798, 134987 (2019).

[13] G. Parisi, The strategy for computing the hadronic mass spectrum, Phys. Rep. 103, 203 (1984).

[14] G. P. Lepage, The analysis of algorithms for lattice field theory, in Proceedings of the 1989 Theoretical Advanced Study Institute in Elementary Particle Physics, From Actions to Answers, edited by T. DeGrand and D. Toussaint (World Scientific, Singapore, 1989), pp. 97-120, 10.1142/ 0971.

[15] T. Iritani, S. Aoki, T. Doi, T. Hatsuda, Y. Ikeda, T. Inoue, N. Ishii, H. Nemura, and K. Sasaki (HAL QCD Collaboration), Are two nucleons bound in lattice QCD for heavy quark masses? Consistency check with Lüscher's finite volume formula, Phys. Rev. D 96, 034521 (2017).

[16] M. L. Wagman, F. Winter, E. Chang, Z. Davoudi, W. Detmold, K. Orginos, M. J. Savage, and P. E. Shanahan (NPLQCD Collaboration), Baryon-baryon interactions and spin-flavor symmetry from lattice quantum chromodynamics, Phys. Rev. D 96, 114510 (2017).

[17] A. Francis, J. R. Green, P. M. Junnarkar, Ch. Miao, T. D. Rae, and H. Wittig, Lattice QCD study of the $H$ dibaryon using hexaquark and two-baryon interpolators, Phys. Rev. D 99, 074505 (2019).

[18] B. Hörz, D. Howarth, E. Rinaldi, A. Hanlon, C. C. Chang, C. Körber, E. Berkowitz, J. Bulava, M. A. Clark, W. T. Lee, C. Morningstar, A. Nicholson, P. Vranas, and A. WalkerLoud, Two-nucleon $S$-wave interactions at the SU(3) flavorsymmetric point with $m_{u d} \simeq m_{s}^{\text {phys }}$ : A first lattice QCD calculation with the stochastic Laplacian Heaviside method, Phys. Rev. C 103, 014003 (2021).

[19] S. Amarasinghe, R. Baghdadi, Z. Davoudi, W. Detmold, M. Illa, A. Parreno, A. V. Pochinsky, P. E. Shanahan, and M. L. Wagman, A variational study of two-nucleon systems with lattice QCD, arXiv:2108.10835.

[20] P. B. Mackenzie and H. B. Thacker, Evidence against a Stable Dibaryon from Lattice QCD, Phys. Rev. Lett. 55, 2539 (1985).

[21] Y. Iwasaki, T. Yoshie, and Y. Tsuboi, $H$ Dibaryon in Lattice QCD, Phys. Rev. Lett. 60, 1371 (1988).

[22] A. Pochinsky, J. W. Negele, and B. Scarlet, Lattice study of the $H$ dibaryon, Nucl. Phys. B, Proc. Suppl. 73, 255 (1999).

[23] I. Wetzorke, F. Karsch, and E. Laermann, Further evidence for an unstable $H$ dibaryon?, Nucl. Phys. B, Proc. Suppl. 83-84, 218 (2000).

[24] I. Wetzorke and F. Karsch, The $H$ dibaryon on the lattice, Nucl. Phys. B, Proc. Suppl. 119, 278 (2003).

[25] Z.-H. Luo, M. Loan, and X.-Q. Luo, H-dibaryon from lattice QCD with improved anisotropic actions, Mod. Phys. Lett. A 22, 591 (2007).

[26] Z.-H. Luo, M. Loan, and Y. Liu, Search for the $H$ dibaryon on the lattice, Phys. Rev. D 84, 034502 (2011).

[27] S. R. Beane, E. Chang, W. Detmold, B. Joo, H. W. Lin, T. C. Luu, K. Orginos, A. Parreño, M. J. Savage, A. Torok, and A. Walker-Loud (NPLQCD Collaboration), Evidence for a Bound $H$ Dibaryon from Lattice QCD, Phys. Rev. Lett. 106, 162001 (2011).

[28] S. R. Beane et al., Present constraints on the $H$-dibaryon at the physical point from lattice QCD, Mod. Phys. Lett. A 26, 2587 (2011.

[29] S. R. Beane, E. Chang, W. Detmold, H. W. Lin, T. C. Luu, K. Orginos, A. Parreño, M. J. Savage, A. Torok, and A. Walker-Loud (NPLQCD Collaboration), The deuteron and exotic two-body bound states from lattice QCD, Phys. Rev. D 85, 054511 (2012).

[30] S. R. Beane, E. Chang, S. D. Cohen, W. Detmold, H. W. Lin, T. C. Luu, K. Orginos, A. Parreño, M. J. Savage, and A. Walker-Loud (NPLQCD Collaboration), Light nuclei and hypernuclei from quantum chromodynamics in the limit of SU(3) flavor symmetry, Phys. Rev. D 87, 034506 (2013).

[31] T. Inoue, N. Ishii, S. Aoki, T. Doi, T. Hatsuda, Y. Ikeda, K. Murano, H. Nemura, and K. Sasaki (HAL QCD Collaboration), Baryon-baryon interactions in the flavor $\mathrm{SU}(3)$ limit from full QCD simulations on the lattice, Prog. Theor. Phys. 124, 591 (2010).

[32] T. Inoue, N. Ishii, S. Aoki, T. Doi, T. Hatsuda, Y. Ikeda, K. Murano, H. Nemura, and K. Sasaki (HAL QCD Collaboration), Bound $H$ Dibaryon in Flavor SU(3) Limit of Lattice QCD, Phys. Rev. Lett. 106, 162002 (2011).

[33] T. Inoue, S. Aoki, T. Doi, T. Hatsuda, Y. Ikeda, N. Ishii, K. Murano, H. Nemura, and K. Sasaki (HAL QCD Collaboration), Two-baryon potentials and $H$-dibaryon from 3 flavor lattice QCD simulations, Nucl. Phys. A881, 28 (2012).

[34] K. Sasaki et al. (HAL QCD Collaboration), $\Lambda \Lambda$ and $\mathrm{N} \Xi$ interactions from lattice QCD near the physical point, Nucl. Phys. A998, 121737 (2020).

[35] M. Peardon, J. Bulava, J. Foley, C. Morningstar, J. Dudek, R. G. Edwards, B. Joó, H.-W. Lin, D. G. Richards, and 
K. J. Juge (Hadron Spectrum Collaboration), Novel quarkfield creation operator construction for hadronic physics in lattice QCD, Phys. Rev. D 80, 054506 (2009).

[36] Preliminary results were presented in Ref. [37].

[37] A. Hanlon, A. Francis, J. Green, P. Junnarkar, and H. Wittig, The $H$ dibaryon from lattice QCD with SU(3) flavor symmetry, Proc. Sci., LATTICE2018 (2018) 081.

[38] M. Bruno et al., Simulation of QCD with $N_{f}=2+1$ flavors of nonperturbatively improved Wilson fermions, J. High Energy Phys. 02 (2015) 043.

[39] See Supplemental Material at http://link.aps.org/supplemental/ 10.1103/PhysRevLett.127.242003, which also includes Refs. [40-51], for details on the lattice methods, additional discussion of the determination and analysis of the spectrum, analysis of two ensembles with a quenched strange quark, and the resampled spectrum data.

[40] M. Lüscher and S. Schaefer, Lattice QCD with open boundary conditions and twisted-mass reweighting, Comput. Phys. Commun. 184, 519 (2013).

[41] J. Bulava and S. Schaefer, Improvement of $N_{f}=3$ lattice QCD with Wilson fermions and tree-level improved gauge action, Nucl. Phys. B874, 188 (2013).

[42] M. Bruno, T. Korzec, and S. Schaefer, Setting the scale for the CLS $2+1$ flavor ensembles, Phys. Rev. D 95, 074504 (2017).

[43] M. Lüscher, Properties and uses of the Wilson flow in lattice QCD, J. High Energy Phys. 08 (2010) 071; 03 (2014) 092(E).

[44] P. Fritzsch, F. Knechtli, B. Leder, M. Marinkovic, S. Schaefer, R. Sommer, and F. Virotta, The strange quark mass and Lambda parameter of two flavor QCD, Nucl. Phys. B865, 397 (2012).

[45] C. Morningstar and M. J. Peardon, Analytic smearing of SU(3) link variables in lattice QCD, Phys. Rev. D 69, 054501 (2004).

[46] B. Hörz and A. Hanlon, Two- and Three-Pion FiniteVolume Spectra at Maximal Isospin from Lattice QCD, Phys. Rev. Lett. 123, 142002 (2019).

[47] C. Morningstar, J. Bulava, J. Foley, K. J. Juge, D. Lenkner, M. Peardon, and C. H. Wong, Improved stochastic estimation of quark propagation with Laplacian Heaviside smearing in lattice QCD, Phys. Rev. D 83, 114505 (2011).

[48] J. R. Green, A. D. Hanlon, P. M. Junnarkar, and H. Wittig, Continuum limit of baryon-baryon scattering with SU(3) flavor symmetry, Proc. Sci. LATTICE2021 (2021) 294.

[49] L. Meng and E. Epelbaum, Two-particle scattering from finite-volume quantization conditions using the plane wave basis, J. High Energy Phys. 10 (2021) 051.

[50] Z. T. Draper and S. R. Sharpe, Applicability of the twoparticle quantization condition to partially-quenched theories, Phys. Rev. D 104, 034510 (2021).

[51] The HDF Group, Hierarchical data format, version 5, 19972021, https://www.hdfgroup.org/HDF5/.

[52] J. J. de Swart, The octet model and its Clebsch-Gordan coefficients, Rev. Mod. Phys. 35, 916 (1963); 37, 326(E) (1965).

[53] M. Lüscher and U. Wolff, How to calculate the elastic scattering matrix in two-dimensional quantum field theories by numerical simulation, Nucl. Phys. B339, 222 (1990).
[54] B. Blossier, M. Della Morte, G. von Hippel, T. Mendes, and R. Sommer, On the generalized eigenvalue method for energies and matrix elements in lattice field theory, J. High Energy Phys. 04 (2009) 094.

[55] M. Lüscher, Two-particle states on a torus and their relation to the scattering matrix, Nucl. Phys. B354, 531 (1991).

[56] K. Rummukainen and S. A. Gottlieb, Resonance scattering phase shifts on a non-rest-frame lattice, Nucl. Phys. B450, 397 (1995).

[57] R. Briceño, Z. Davoudi, and T. Luu, Two-nucleon systems in a finite volume: Quantization conditions, Phys. Rev. D 88, 034502 (2013).

[58] R. A. Briceño, Two-particle multichannel systems in a finite volume with arbitrary spin, Phys. Rev. D 89, 074507 (2014).

[59] C. Körber, E. Berkowitz, and T. Luu, Renormalization of a contact interaction on a lattice, arXiv:1912.04425.

[60] G. R. Farrar, Stable sexaquark, arXiv:1708.08951.

[61] C. Gross, A. Polosa, A. Strumia, A. Urbano, and W. Xue, Dark matter in the standard model?, Phys. Rev. D 98, 063005 (2018).

[62] G. R. Farrar, A precision test of the nature of dark matter and a probe of the QCD phase transition, arXiv:1805.03723.

[63] E. W. Kolb and M. S. Turner, Dibaryons cannot be the dark matter, Phys. Rev. D 99, 063519 (2019).

[64] S. D. McDermott, S. Reddy, and S. Sen, Deeply bound dibaryon is incompatible with neutron stars and supernovae, Phys. Rev. D 99, 035013 (2019).

[65] J. P. Lees et al. (BABAR Collaboration), Search for a Stable SixQuark State at BABAR, Phys. Rev. Lett. 122, 072002 (2019).

[66] K. Azizi, S. S. Agaev, and H. Sundu, The scalar hexaquark uuddss: A candidate to dark matter?, J. Phys. G 47, 095001 (2020).

[67] G. R. Farrar, Z. Wang, and X. Xu, Dark matter particle in QCD, arXiv:2007.10378.

[68] M. Padmanath, J. Bulava, J. R. Green, A. D. Hanlon, B. Hörz, P. Junnarkar, C. Morningstar, S. Paul, H. Wittig, $H$ dibaryon away from the $\mathrm{SU}(3)_{f}$ symmetric point, Proc. Sci. LATTICE2021 (2021) 459.

[69] P. E. Shanahan, A. W. Thomas, and R. D. Young, Mass of the $H$ Dibaryon, Phys. Rev. Lett. 107, 092004 (2011).

[70] Y. Yamaguchi and T. Hyodo, Quark-mass dependence of the $H$ dibaryon in $\Lambda \Lambda$ scattering, Phys. Rev. C 94, 065207 (2016).

[71] Jülich Supercomputing Centre, JUQUEEN: IBM Blue Gene/Q supercomputer system at the Jülich Supercomputing Centre, J. Large-Scale Res. Facil. 1, A1 (2015).

[72] Jülich Supercomputing Centre, JURECA: Modular supercomputer at Jülich Supercomputing Centre, J. Large-Scale Res. Facil. 4, A132 (2018).

[73] Jülich Supercomputing Centre, JUWELS: Modular tier-0/1 supercomputer at the Jülich Supercomputing Centre, J. Large-Scale Res. Facil. 5, A135 (2019).

[74] R. G. Edwards and B. Joó (SciDAC, LHPC, and UKQCD Collaborations), The CHROMA software system for lattice QCD, Nucl. Phys. B, Proc. Suppl. 140, 832 (2005).

[75] A. Stathopoulos and J.R. McCombs, PRIMME: Preconditioned iterative multimethod eigensolver-methods and software description, ACM Trans. Math. Softw. 37, 1 (2010). 
[76] M. Lüscher and S. Schaefer, OPENQCD, 2012, http://luscher .web.cern.ch/luscher/openQCD/.

[77] D. G. A. Smith and J. Gray, opt_einsum: A Python package for optimizing contraction order for einsum-like expressions, J. Open Source Softw. 3, 753 (2018).

[78] C. Morningstar, SigMonD, https://github.com/andrewhanlon/ sigmond (2021).

[79] C. R. Harris, K. J. Millman, S. J. van der Walt, R. Gommers, P. Virtanen, D. Cournapeau, E. Wieser, J. Taylor, S. Berg,
N. J. Smith, R. Kern et al., Array programming with NUMPY, Nature (London) 585, 357 (2020).

[80] P. Virtanen, R. Gommers, T. E. Oliphant, M. Haberland, T. Reddy, D. Cournapeau, E. Burovski, P. Peterson, J. Weckesser, W. Bright, S. J. van der Walt et al., SCIPY 1.0: Fundamental algorithms for scientific computing in PYTHON, Nat. Methods 17, 261 (2020).

[81] J. D. Hunter, MATPLOTLIB: A 2D graphics environment, Comput. Sci. Eng. 9, 90 (2007). 